\title{
Multimode fiber beam self-cleaning in the anomalous dispersion regime
}

\author{
Y. Leventoux ${ }^{1}$, A. Parriaux ${ }^{2}$, G. Granger ${ }^{1}$, M. Jossent ${ }^{3}$, L. Lavoute ${ }^{3}$, D. Gaponov ${ }^{3}$, M. Fabert ${ }^{1}$, A. Tonello ${ }^{1}$, \\ K. Krupa ${ }^{2}$, A. Desfarges-Berthelemot ${ }^{1}$, V. Kermene ${ }^{1}$, O. Sidelnikov ${ }^{4}$, G. Millot ${ }^{2}$, S. Février ${ }^{1}$, S. Wabnitz ${ }^{4,5}$, and \\ V. Couderc ${ }^{1}$ \\ 1. Université de Limoges, XLIM, UMR CNRS 7252, 123 Avenue A. Thomas, 87060 Limoges, France \\ 2. Université Bourgogne Franche-Comté, ICB UMR CNRS 6303, 9 Avenue A. Savary, 21078 Dijon, France \\ 3. Novae, ZI du Moulin Cheyroux, Aixe sur Vienne, France \\ 4. Novosibirsk State University, Novosibirsk, Russia \\ 5. DIET, Sapienza Università di Roma, Via Eudossiana 18, 00184 Roma, Italy \\ stefan.wabnitz@uniroma1.it
}

\begin{abstract}
We experimentally demonstrate Kerr beam self-cleaning of picosecond pulses in the anomalous dispersion regime of graded-index multimode optical fibers, with threshold power reduced by two orders of magnitude with respect to the normal dispersion regime. (C) 2019 The Author(s) OCIS codes: (190.4370) Nonlinear optics, fibers; (190.3270) Kerr effect; (190.4380) Nonlinear optics, four-wave mixing; (190.5940) Self-action effects
\end{abstract}

There is currently much interest in exploiting multimode optical fibers (MMFs) for future long-distance communications using spatial division multiplexing (SDM) [1]. On the other hand, MMFs are extensively used in short-distance links, e.g., inter and intra-data center networks. In this context, a relevant problem is the effective coupling and stable transport of a diffraction limited optical beam from a single mode (SM) laser source into the fundamental mode of the MMF. Mode coupling limits the MMF length to a few tens of mm before the beam quality is severely degraded. Moreover, in order to increase the pulse energy delivered by fiber amplifiers, MMFs should be used: effective SM propagation can be achieved by gain-guiding [2].

In this work, we experimentally demonstrate, we believe for the first time, that mode coupling can be suppressed in a graded-index (GRIN) MMF, for picosecond pulses at telecom wavelengths (i.e., $1562 \mathrm{~nm}$ ), by exploiting the Kerr nonlinearity of the fiber. In this way, high quality SM beams can be effectively propagated over more than 10 $\mathrm{m}$ of GRIN MMF. Depending on the input spatial coupling conditions (i.e., incidence angle of the beam), we observed that the beam at the fiber output exhibits a very robust spatial reshaping (accompanied with nearly complete intensity correlation) into either the fundamental $\mathrm{LP}_{01}$ or the higher order $\mathrm{LP}_{11}$ mode. This reshaping is analogous to Kerr beam self-cleaning recently observed in the normal group velocity dispersion (GVD) regime [34]. However, quite remarkably, the power threshold is reduced by about two orders of magnitude in the anomalous GVD regime, thanks to optimized beam coupling from a SM fiber laser.

The experimental set-up to observe spatial self-cleaning was based on the dispersive broadening (and chirping) of the full-width at half-maximum (FWHM) duration fiber laser pulses from 1 ps to 90 ps, with $2 \mathrm{~nm}$ (at $-3 \mathrm{~dB}$ ) spectral bandwidth. The fiber laser wavelength was $1562 \mathrm{~nm}$, with $1 \mathrm{MHz}$ repetition rate. The beam spot was of Gaussian shape, and $\sim 20 \mu \mathrm{m}$ in diameter full width at $1 / \mathrm{e}^{2}$ in intensity, corresponding to optimized free-space coupling efficiency (up to $80 \%$ ) into the MMF. Fig. 1 shows the self-cleaning of the output near-field intensity patterns. For peak powers above $5 \mathrm{~W}$, the speckled output intensity pattern resulting from the coherent superposition of the multimode field self-cleans into a bell-shaped beam, with negligible multimode field background.

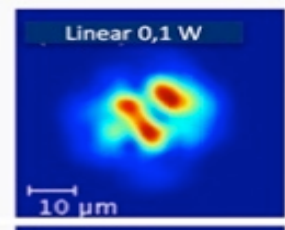

$66 \mathrm{~W}$

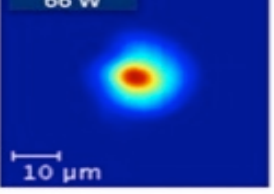

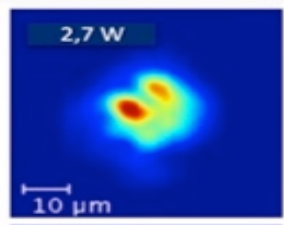
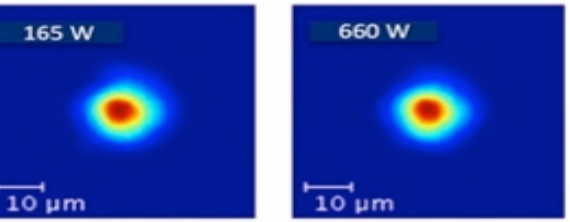
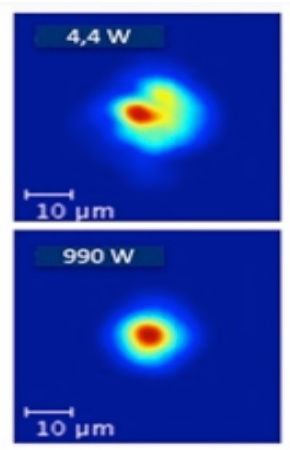
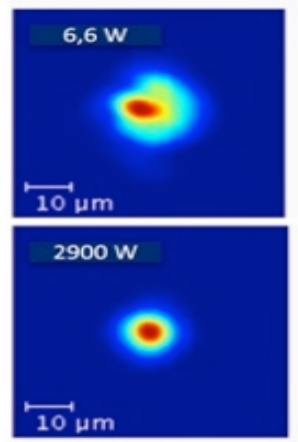

Fig. 1 Near field intensity patterns at the fiber output recorded for increasing input peak powers, leading to self-cleaning of the $\mathrm{LP}_{01}$ fundamental mode of the GRIN MMF. 
The $12 \mathrm{~m}$ long fiber was loosely coiled on the table, forming rings of $\sim 15 \mathrm{~cm}$ diameter. The fiber had a circular core of $25 \mu \mathrm{m}$ radius, with a core-cladding index difference of 0.015 , for a 0.2 numerical aperture. We verified from Figure 2 that our input conditions lead to $99.9 \%$ of the pulse energy coupled into the first three radially symmetric modes only.
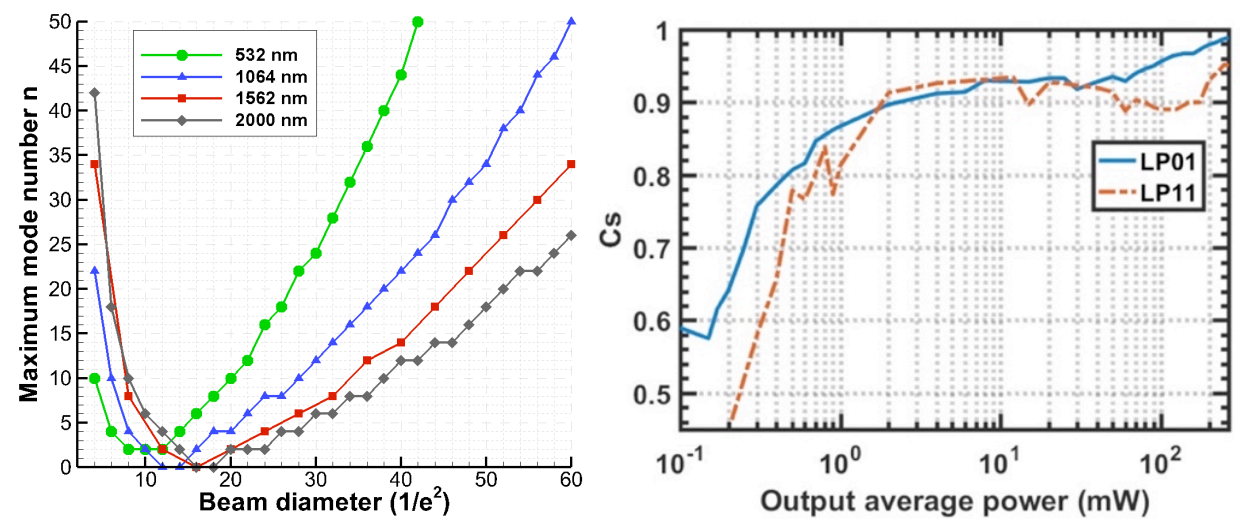

Fig. 2. Left: maximum number of excited radial modes vs. input beam diameter, for different wavelengths; Right: Intensity correlation $\mathrm{C}_{\mathrm{S}}$ vs. output average power for $\mathrm{LP}_{01}$ (solid curve) and $\mathrm{LP}_{11}$ (dashed curve) modes.

We measured the intensity correlation $\mathrm{C}_{\mathrm{S}}$ of the near field pattern of the output beam with respect to the fundamental mode $\left(\mathrm{LP}_{01}\right)$, numerically obtained with a mode solver. Fig. 2, right, shows that the intensity correlation $\mathrm{C}_{\mathrm{S}}$ with the fundamental mode keeps monotonically increasing as the input peak power grows larger, until reaching more than $90 \%$ at high powers. Figs. 1-2 show that the process of pulse beam self-cleaning in the anomalous GVD regime is much more efficient than in previous experiments carried out in the normal GVD regime, in two respects. First, the threshold for self-cleaning is reduced by about two orders of magnitude (from $0.5 \mathrm{~kW}$ [3] down to 4-5 W) for a GRIN fiber length of about $12 \mathrm{~m}$. Second, nearly complete intensity correlation with the fundamental mode is achieved at the fiber output, in contrast with experiments in the normal GVD regime and with a wider input beam, where large residual background was observed around the self-cleaned beam [3]. Figs 1-2 show that, in spite of coupling more than $90 \%$ of the input beam energy into the fundamental mode of the GRIN MMF, after propagation over $12 \mathrm{~m}$ the output beam quality is strongly degraded, owing to linear mode coupling with higher-order modes. Here the Kerr effect acts as a stabilizer of beam coupling from a SM fiber laser into a GRIN MMF, by preventing energy flow out of the fundamental mode. This occurs because of the nonlinear nonreciprocity of mode coupling process [3]: the nonlinear contribution to the propagation constant of the fundamental mode brings out of phase-matching the random linear mode coupling processes.

In our experiments, by varying the position and tilt of the Gaussian laser beam launched at the GRIN-MMF input face, as well as the cleave angle of the fiber input, we observed self-cleaning into the LP $_{11}$ mode, with a peak power threshold of $50 \mathrm{~W}$, about two orders of magnitude lower than for $\mathrm{LP}_{11}$ self-cleaning in the normal GVD regime [5].

To conclude, we have experimentally shown that Kerr beam self-cleaning in GRIN-MMFs can occur with extreme efficiency in the anomalous dispersion regime. The threshold for beam self-cleaning is reduced by about two orders of magnitude with respect to previous experiments in the normal dispersion regime. These results are significant for applications of MMFs in many fields, e.g., data transport in data-center communications, secure quantum optical communications, nonlinear medical imaging, high-energy laser beam delivery, multimode fiber lasers and amplifiers operating in the near-infrared, and SDM transmissions.

We acknowledge support from: EU Horizon 2020 research and innovation program - European Research Council (ERC) grant No. 740355; Agence Nationale de la Recherche (ANR) (Broadband Infrared Supercontinuum BISCOT ANR-16-CE080031); Région Nouvelle Aquitaine (FLOWA); Direction Générale de l'Armement (DGA); french program "Investissements d'Avenir", project ISITE-BFC (contract ANR-15-IDEX-0003). K.K. thanks the Marie-Skłodowska-Curie grant No. 713694; O.S. thanks the Russian Ministry of Science and Education (Grant 14.Y26.31.0017).

\section{References}

[1] S. Mumtaz et al. "Nonlinear propagation in multimode and multicore fibers: generalization of the Manakov equations," J. Lightwave. Technol. 31398 (2013).

[2] M.E. Fernmann et al., "Single-mode amplifiers and compressors based on multimode fibers," US Patent 5818630 (1998).

[3] K. Krupa et al., "Spatial beam self-cleaning in multimode fiber," Nat. Photon. 11, p. 237 (2017).

[4] Z. Liu et al., "Kerr self-cleaning of femtosecond-pulsed beams in graded-index multimode fiber," Opt. Lett. 41, p. 3675 (2016).

[5] E. Deliancourt et al., "Kerr beam auto-selection on a low order mode in graded-index multimode fiber," arXiv: 1807.00563 (2018). 